Preface

\section{Orbital Reconstruction}

\author{
Sameep Kadakia, MD ${ }^{1}$
}

${ }^{1}$ Department of Plastic and Reconstructive Surgery, Boonshoft

School of Medicine at Wright State University, Dayton, Ohio

Semin Plast Surg 2019;33:83-84.

Orbital reconstruction poses a true challenge for reconstructive surgeons owing to the three-dimensional anatomy of the orbit, proximity to the brain and sinonasal tract, as well as the delicate interface between bone and soft tissue. A thorough understanding of both form and function allows successful reconstruction of this unique region. A defect-driven approach coupled with an understanding of the patient's overall health can guide the reconstructive surgeon in choosing viable options. Orbital reconstruction truly lends itself to the art and skill of the surgeon, as it spans the gamut from trauma repair to resurfacing of exenteration defects to skull base reconstruction. Soft-tissue techniques utilized for eyelid reconstruction are equally challenging due to the propensity of the periorbital structures to readily fibrose and compromise ideal function.

An important facet of orbital reconstruction is the ability to call for multidisciplinary collaboration. This unique region not only poses significant challenge, but also fosters the opportunity for surgeons from multiple specialties to come together and draw on one another's strengths in order to gen-

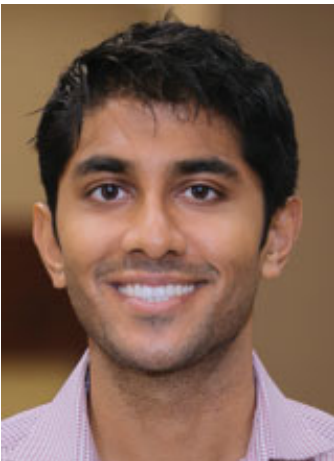

Sameep Kadakia, MD erate an optimal outcome for the patient.

In this issue, we will begin by studying the surgical anatomy of the orbit, after which we will discuss principles of trauma management and eyelid repair. Several sections in this volume will also be dedicated to orbital exenteration, as well as the regional, microvascular, and prosthetic reconstruction options. Lastly, posttraumatic defects of the nasoorbitoethmoid region will also be examined.

It is with great excitement that I present this issue of Seminars in Plastic Surgery. It has been an honor working with many talented surgeons in compiling this collection of our work.
Copyright $\odot 2019$ by Thieme Medical Publishers, Inc., 333 Seventh Avenue, New York, NY 10001, USA. Tel: +1(212) 584-4662.
DOI https://doi.org/ 10.1055/s-0039-1685210. ISSN $1535-2188$. 